\title{
Percepção do Adulto-Jovem sobre o Envelhecer
}

\author{
Jéssica Santos Filadelfo ${ }^{1}$; Aldrina da Silva Confessor Cândido ${ }^{2}$
}

\begin{abstract}
Resumo: Este estudo teve como objetivo conhecer a percepção de adultos-jovens sobre o envelhecer. A pesquisa foi do tipo descritiva com abordagem qualitativa e procedimento de pesquisa de campo com discentes de uma instituição de nível superior no município de Vitória da Conquista-BA. A análise dos dados foi através da técnica da Análise de Conteúdo, que consiste em um conjunto de técnicas de julgamento das comunicações que utiliza procedimentos sistemáticos e objetivos de descrição de conteúdo. A maioria dos entrevistados definiu o envelhecimento abordando principalmente os aspectos biológicos e cronológicos. Entender o processo de envelhecimento e a percepção de cada um sobre esse assunto é importante, e o quanto mais cedo possível, melhor, não somente para ampliar os conhecimentos dos processos degenerativos associados a idade, mas também para desenvolver estratégias que amenizem os efeitos físicos e mentais decorrente da passagem dos anos.
\end{abstract}

Palavras-chave: envelhecimento, idosos, autopercepção.

\section{Perception of the Younger Adult on Aging}

\begin{abstract}
This study aimed to know the perception of young adults on aging. The research was descriptive with qualitative approach and field research procedure with students of a higher education institution in the city of Vitoria da Conquista/BA. Data analysis was through the content analysis technique, which consists of a set of techniques of trial communication using systematic procedures and content description goals. Most respondents defined the aging mainly addressing the biological and chronological. Understand the process of aging and perception of each on this issue is important, and as soon as possible, the better, not only to increase knowledge of the degenerative processes associated with age, but also to develop strategies that mitigate the physical effects and mental resulting from the passage of years.
\end{abstract}

Keywords: aging, elderly, selfperception.

\section{Introdução}

O envelhecimento é um processo biológico progressivo caracterizado pelos efeitos ou consequências da passagem do tempo no organismo, variável de indivíduo para indivíduo a depender de fatores como estilo de vida, condições socioeconômicas e doenças crônicas (FECHINE; TROMPIERI, 2012). Pode ser definido em várias perspectivas, como em termos biológicos, cronológicos, sociais, intelectuais, econômicos e funcionais.

\footnotetext{
1 Aluna do Curso de Enfermagem da Faculdade Independente do Nordeste - FAINOR, Vitória da Conquista - BA. E-mail: jeufiladelfo@gmail.com

${ }^{2}$ Docente do Curso de Enfermagem da Faculdade Independente do Nordeste - FAINOR, doutoranda em Humanidades e Arte com ênfase em Ciências da Educação. Vitória da Conquista - BA. E-mail: aldrina@fainor.com.br
} 
Id on Line Revista Multidisciplinar e de Psicologia

Id on Line Multidisciplinary Journal and Psycology

Biologicamente consiste em uma redução funcional contínua nos diversos sistemas: neurológico, respiratório, circulatório, digestivo, geniturinário e musculoesquelético (KAUFFMAN; JACKSON, 2001). Quanto ao aspecto social e econômico, acontece de acordo com o momento histórico-cultural e na medida em que o indivíduo deixa de ser produtivo; e, em termos funcionais quando este passa a necessitar de auxilio para a execução de suas necessidades básicas (PASCHOAL, 2002).

Além das alterações fisiológicas, o envelhecimento também propicia outras mudanças. Do ponto de vista psicológico é uma fase de maturidade, de sabedoria adquirida com o decorrer dos anos e da experiência de vida acumulada, contudo é marcado por perdas acentuadas e algumas vezes sentimentos de isolamento, baixa estima e depressão, além do agravamento de problemas de saúde em geral (SILVA et al., 2005).

Este processo também contribui com o aparecimento de inúmeras doenças, como: osteoartose; osteoporose; contratura muscular, que acarreta deformidades posturais; déficit de marcha; diminuição da força muscular e da mobilidade corporal; diminuição da capacidade de expansão do gradil costal e outros que limitam o movimento corporal, geralmente acompanhado de quadro álgico (CÓTICA, 2011).

Atualmente é notório o crescimento do número de idosos em todo o mundo. No Brasil a população idosa corresponde a $7,4 \%$ da população, de acordo com dados do último censo demográfico (IGBE, 2010). Esse aumento pode ser atribuído à redução das taxas de mortalidade infantil, migração da população rural, melhores condições de saúde e saneamento somadas aos avanços tecnológicos da medicina na prevenção e tratamento de várias doenças. Além disso, é necessário qualidade de vida adequada e bem-estar no dia-a-dia dos mais velhos ampliando as suas oportunidades sociais e econômicas.

O envelhecimento pode ser entendido como um processo dinâmico que ocorre ao longo do tempo envolvendo mudanças constantes em diversos aspectos da vida e deve ser compreendido de acordo com a percepção individual e das experiências do próprio indivíduo.

A percepção de uma pessoa sobre si corresponde a um processo de reconhecimento e identificação dos estímulos do ambiente em redor. A auto percepção do envelhecimento tem sido sugerida como um preditor independente da capacidade funcional e da longevidade na velhice (BARKER et al., 2007). Sua relação com o equilíbrio psicológico está pautada com 
Id on Line Revista Multidisciplinar e de Psicologia

Id on Line Multidisciplinary Journal and Psycology

aspectos do funcionamento psíquico, como a satisfação e a qualidade de vida, a solidão e a depressão (YASINE, 2011).

Conforme envelhecemos continuamos a trabalhar e a redefinir nossos autoconceitos em busca da concretização de projetos pessoais, e a compreensão de experiências passadas podem resultar na obtenção de sentimentos de autoridade, controle e domínio sobre as nossas vidas (HANSSON e STROE, 2007).

A percepção do envelhecimento e a auto percepção da idade são, em parte, modelados pelas interações dinâmicas com sistemas sociais e culturais, que enquadram a nossa compreensão da idade e do envelhecimento no contexto de uma população envelhecida com uma experiência de vida em constante mutação (YASSINE, 2011). O autor destaca que a percepção de uma pessoa sobre si mesma corresponde a um processo de detecção e identificação dos estímulos do ambiente em redor, e nossa percepção não é apenas uma reprodução fotográfica da realidade, mas sim uma construção personalizada.

Shin, Kim e Kim (2002) defende a ideia de que a informação veiculada pela percepção em relação ao envelhecer é integrada em seis dimensões pessoais básicas: a mudança e controle sobre o próprio corpo, a percepção da idade, o espaço físico, o rumo da vida e a reestruturação de relações significativas.

Nesse contexto e sob tal perspectiva, esse trabalho justificou-se devido à necessidade de saber como o adulto jovem enxerga o processo do envelhecimento mental e físico. Para entender os fenômenos causados na mente humana decorrentes do envelhecimento, é fundamental o estudo da percepção desse processo nos adultos-jovens, a fim de conhecer suas expectativas de vida futura, como pensam e encaram a velhice e seus desafios, e as intervenções possíveis de forma a melhorar a qualidade de vida. Assim, a questão norteadora desse estudo é: qual a percepção dos adultos-jovens sobre o processo de envelhecimento?

Para responder a questão norteadora, o presente estudo tem por objetivo geral: conhecer a percepção do adulto-jovem sobre o envelhecer, e por objetivos específicos: conceituar velhice/envelhecimento a partir da visão do adulto- jovem; descrever a visão do adulto- jovem sobre o envelhecimento e redimensionar as posturas do adulto- jovem em relação ao processo de envelhecer. 
Id on Line Revista Multidisciplinar e de Psicologia

Id on Line Multidisciplinary Journal and Psycology

\section{Metodologia}

O estudo foi descritivo com abordagem qualitativa, já que visou compreender a percepção de adultos-jovens sobre as questões relativas ao envelhecimento, descrevendo assim as suas representações. Quanto ao procedimento foi uma pesquisa de campo, já que a coleta de dados foi realizada numa Instituição de Ensino Superior (IES) no município de Vitória da Conquista-BA, situada na região Sudoeste da Bahia e considerada a terceira maior cidade do Estado, com uma população de 306.866 mil habitantes, de acordo com dados do IBGE (2010). A cidade consiste em um polo econômico regional, e destaca-se no comércio, indústria e mais fortemente na prestação de serviços, notadamente os relacionados à área de saúde e de educação.

Os participantes do estudo foram 6 adultos-jovens, 03 do sexo feminino e 03 do sexo masculino, com faixa etária entre 20 e 30 anos de idade, residentes no município e discentes da referida instituição.

Como instrumento de coleta de dados utilizou-se um questionário com questões semiestruturadas, inicialmente constituído de questões voltadas para caracterização da amostra, como sexo, idade, profissão/ocupação e curso; e a segunda parte correspondente aos objetivos da pesquisa.

A análise dos dados foi através da técnica da Análise de Conteúdo (AC), que consiste em um conjunto de técnicas de julgamento das comunicações que utiliza procedimentos sistemáticos e objetivos de descrição de conteúdo (BARDIN, 2009). Ou seja, a análise de conteúdo permite analisar o que foi dito nas entrevistas ou observado pelo pesquisador, de modo a classificá-los em temas ou categorias que auxiliam na compreensão do que está por trás dos discursos

De acordo com Godoy (2005) a análise de conteúdo é composta por três fases fundamentais, a pré-análise, a exploração do material e os resultados. A pré-análise é uma fase de organização em que estabelece um esquema de trabalho que deve ser preciso, com procedimentos bem definidos e flexíveis, e envolve contato inicial com o material coletado. $\mathrm{Na}$ fase de exploração foi realizada a leitura do material, adotando procedimentos de codificação, classificação e categorização. Na fase de resultados, realizou-se a análise dos dados, a 
formulação de hipóteses e a elaboração de categorias para orientar a interpretação e a preparação do conteúdo final.

A pesquisa foi submetida ao Comitê de Ética e Pesquisa, sendo aprovada mediante parecer $n^{0}$. 1.585.663. Foi solicitado autorização à Instituição de Ensino Superior para a realização pesquisa, sendo os dados coletados no mês de Agosto de 2016. Todos os participantes tiveram garantia de sigilo e assinaram o Termo de Consentimento Livre e Esclarecido (TCLE).

\section{Resultados e Discussão}

Entre os seis participantes da pesquisa, três eram do sexo masculino e três do sexo feminino; apresentavam idade entre 20 e 30 anos, sendo um estudante do curso de direito, um do curso de enfermagem, dois do curso de odontologia e dois cursavam fisioterapia. Para conhecer a visão do adulto-jovem sobre o processo de envelhecimento, os resultados do presente estudo foram divididos em três categorias distintas.

\section{Categoria I - O Adulto Jovem e sua Concepção de Envelhecimento}

Existem vários conceitos para definir o envelhecimento, embora a maioria dos autores ressaltem quase sempre as mesmas características. Veras (2002) o define como um conjunto de alterações estruturais e funcionais do organismo que se acumula de forma progressiva devido a vários fatores em especial em decorrência da idade dos indivíduos.

O envelhecimento é comum a todos os seres humanos, contudo há particularidades ao envelhecer, ou seja, o envelhecimento é um processo individual com características comuns a todos. Pode ser definido em várias perspectivas, como em termos biológicos, cronológicos, sociais, intelectuais, econômicos e funcionais.

A maioria dos entrevistados definiu o envelhecimento abordando principalmente os aspectos biológicos e cronológicos. Nas falas dos participantes da pesquisa fica evidente tal concepção temporal e biológica do envelhecimento, já que estes citam:

“Amadurecimento tanto mental como físico.” (E01)

“Degeneração gradativa das células.” (E02) 
Corroborando com as definições de grande parte dos entrevistados, De Vitta (2000) destaca que algumas alterações biológicas do organismo resultam naturalmente do envelhecimento normal, e Netto (2002) enfatiza que o envelhecimento biológico é universal e comum em todos os seres vivos animais.

Ainda nessa perspectiva, Kaufman e Jackson (2001), ao conceituar o envelhecimento, o define como um processo dinâmico e progressivo de ordem natural do ser humano com intensidade variável conforme cada organismo, ocasionando mudanças morfológicas, funcionais e bioquímicas.

Moraes et al. (2009) ressalta que há evidências de que o processo de envelhecimento ocorre devido a vários fatores e depende da programação genética e das mudanças que acontecem em nível celular-molecular, sendo acompanhado por diminuição funcional e progressiva de vários sistemas orgânicos. Além disso, os autores observam que os sinais de deficiências funcionais aparecem de forma discreta durante toda a vida e não podem ser considerados como doença.

\section{Categoria II - O Adulto Jovem e os Aspectos Positivos e Negativos do Envelhecimento}

Em relação aos aspectos positivos do processo de envelhecimento, de acordo com a pesquisa, observou-se que apenas dois entre os participantes responderam não haver pontos positivos. No entanto, os demais ressaltaram aspectos como conhecimentos adquiridos, amadurecimento e muitas experiências vividas, além de mais tempo para descansar e se divertir. Ressaltou-se, até mesmo, o aspecto do aumento da credibilidade frente à sociedade.

“Conhecimento, amadurecimento e confiança." (E01)

“Tempo para descansar, para sair, alguns se divertem.” (E02)

“Ter uma boa saúde para viver e saber que tudo que passou valeu a pena.” (E03)

“Viver experiências e passar credibilidade”. (E06) 
Id on Line Revista Multidisciplinar e de Psicologia

Id on Line Multidisciplinary Journal and Psycology

Ao comparar as respostas obtidas nesse estudo, pode-se perceber que a visão deles é bem próxima a de pessoas idosas, como observa-se na pesquisa realizada por Cótica (2011), com o objetivo de avaliar a percepção de envelhecimento de 16 idosos com idades entre 75 e 89 anos. As respostas atribuídas ao sentido da velhice foram agrupadas em aspectos positivos e negativos, e o autor notou que dez entre os dezesseis idosos incluíram nos aspectos positivos sentimentos de respeito, importância, experiência e realização com a velhice.

É importante ressaltar, ainda, a atribuição de novos conceitos ao envelhecer, conforme relata Dutra (2015), até mesmo deixando de considerar esta fase como uma última etapa da vida, passando esta a ser vista como um estilo de vida, no qual surge oportunidades de viver experiências não vivenciadas anteriormente; o que pode, na visão não só do idoso mas do adulto-jovem, ser considerada uma maneira de protelar o envelhecimento.

Confirmando os aspectos positivos destacados pelos participantes, Cótica (2011) enfatiza que o envelhecimento possibilita ao indivíduo a aquisição de sabedoria, experiência, sobriedade e ponderação, de modo que ele também propicia o amadurecimento do ser. Nesse sentido, Motta (2004) ressalta que o envelhecimento é reflexo de inter-relações sociais e individuais, oriundas da educação, trabalho e experiência de vida.

Para Neri (2001) a maturidade traz não só a excelência em termos de conhecimento, mas também a capacidade de se tornar sábio, ao passo que é evidente o aspecto vivencial exigido para se determinar a sabedoria do ser humano.

Dentre os aspectos negativos citados em relação ao envelhecimento, observou-se que do total, quatro entrevistados mencionaram a preocupação com saúde e a susceptibilidade a doenças, limitações físicas e a solidão, conforme relatos abaixo.

"Os problemas de saúde que podem se agravar e a imunidade vai ficando baixa." (E02)

“Vulnerável a vários tipos de doença.” (E03)

“Doenças, degeneração muscular e cansaço." (E04)

“Doenças típicas da idade, dependência e solidão/abandono.” (E05)

Percepções semelhantes foram verificadas na pesquisa de Cótica (2011), em que 37,5\% dos entrevistados enfatizaram principalmente a preocupação com a perda da saúde física e $12,5 \%$ relataram a solidão. 
Estudos epidemiológicos vêm demonstrando que doenças e limitações não são consequências inevitáveis do envelhecimento, elas dependerão do acesso que o indivíduo tem durante a vida aos serviços preventivos, que o orientam para a redução de fatores de risco e levam à adoção de hábitos de vida saudáveis (RIBEIRO, ALVES e MEIRA, 2009). Conforme Bretas (2003) também irão depender da visão de mundo da sociedade em que está inserido, e das condições socioeconômicos do próprio indivíduo.

\section{Categoria III - O Adulto Jovem e a Construção de uma Velhice de Qualidade}

Quando questionados sobre como é envelhecer com qualidade, quase todos (05) participantes citaram algo relacionado a saúde, dentre outros aspectos como família, independência e satisfação. O participante que não citou a saúde diretamente destacou a importância da dieta adequada e prática de exercícios físicos, que não deixam de contribuir também com esse aspecto.

\footnotetext{
“Aproveitar todos os momento ao máximo, tentar ser feliz, buscar satisfação, sem esquecer é claro da saúde. ”(E01)

“É ter boa saúde, alimentação saudável, sem estresse e problemas. ”(E02)

“Envelhecer com saúde e família por perto.” (E03)

"É viver próximo de quem gosta, desfrutar de saúde física, mental e social, se sentir amado e com sentimento de dever cumprido." (E05)

“Envelhecer com o máximo de saúde e independência.”(E06)
}

Com a pesquisa, observa-se que hoje em dia começa cada vez mais cedo as preocupações com cuidados relacionados a saúde, fator essencial tanto para a qualidade de vida atual como a influencia que representa na vida futura, ou seja, na "velhice".

Foi questionado também aos participantes se, de alguma forma, há algum preparo por parte deles para essa etapa da vida e como se preparam. Verificou-se que metade dos participantes responderam que não se preparam, e somente um afirmou que sim.

"Mais ou menos, tem momentos que me preocupo mais em outros sou mais relapsa." (E01) 
Um estudo desenvolvido por Ribeiro, Cendoroglo e Lemos (2015, p.81) buscou "compreender os hábitos de vida que os idosos tiveram quando jovens e a influencia destes em sua saúde e envelhecimento". Um dos entrevistados, idoso, relatou que "a juventude se desperdiça em prazeres prejudiciais à saúde, incluindo álcool e tabaco. A grande maioria não se prepara para o futuro, não busca o cuidado com a saúde" (RIBEIRO, CENDOROGLO e LEMOS, 2015, p.93). Ficou evidenciado, ao longo do referido estudo, o quão importante é, que o jovem, perceba desde cedo que tem o poder de mudar e de guiar a sua saúde.

De acordo com Assis e Araújo (2004) as mudanças fisiológicas do envelhecimento combinadas com a falta de atividades físicas podem acarretar em processos patológicos que podem levar o idoso a uma perda progressiva de autonomia e independência. Assim, pessoas que se mantém ativas ao longo da vida apresentam ganhos na saúde, com maior autonomia e independência (CRESS et al, 1999).

Além disso, atividades físicas regulares influenciam beneficamente as capacidades funcionais e a qualidade de vida do indivíduo, bem como contribui com a saúde mental de jovens e idosos (SHEPHARD, 2003). O autor ainda ressalta que a atividade física pode aumentar de 6 a 10 anos a expectativa de vida e também a qualidade.

\section{Considerações Finais}

Observou-se nesse estudo que metade das pessoas entrevistadas, mesmo ainda jovens, já pensam sobre o seu envelhecimento, prevalecendo no seu imaginário uma concepção biológica e cronológica do processo de envelhecer.

$\mathrm{Na}$ visão dos adultos-jovens, os aspectos positivos mais presentes no envelhecimento estão associados aos conhecimentos adquiridos ao longo dos anos, ao amadurecimento e as experiências vividas, evidenciando que a vida é um constante aprendizado, e que há a percepção de que o passar do tempo nos torna pessoas mais sábias. 
A preocupação com a saúde e a predisposição a doenças foram os aspectos negativos mais relatados. Contudo, a adoção de hábitos de vida saudável e de práticas preventivas é entendida, por esses adultos jovens, como fatores que contribuem de forma considerável para um envelhecimento com maior qualidade. Estes também percebem que as atividades físicas regulares influenciam positivamente nas capacidades funcionais e na qualidade de vida, contribuindo para a saúde tanto agora como no futuro, apesar de, na sua maioria não se prepararem para o envelhecimento.

Diante do exposto, conclui-se que entender o processo de envelhecimento e a percepção de cada um sobre esse assunto é importante, e o quanto mais cedo possível, melhor, não somente para ampliar os conhecimentos dos processos degenerativos associados a idade, mas também para desenvolver estratégias que amenizem os efeitos físicos e mentais decorrente da passagem dos anos.

Isso pode estimular a busca de estratégias para garantir que a fase final do ciclo de vida seja contemplada de forma autônoma e com maior qualidade, pois o processo de envelhecimento não depende somente da nossa condição genética, mas de hábitos que adotamos ao longo da nossa vida. Sugere-se considerar tratar o envelhecimento desde a mais tenra idade visando reforçar a cultura para o envelhecimento ativo e saudável.

\section{Referências}

ASSIS, M. E.; ARAÚJO, T.D. Atividade e postura corporal. In: A.L. SALDANHA; C.P. Caldas (Ed), Saúde do Idoso: a arte de cuidar. 2a edição. Rio de janeiro: Ineterciência, p.8386, 2004.

BARDIN, L. Análise de Conteúdo. Lisboa, Portugal: Edições 70, LDA, 2009.

BARKER, M.; O'HANLON, A.; McGEE, H. M.; HICKEY A.; CONROY, R. M. Crosssectional alidation of the Aging Perceptions Questionnaire: A multidimensional instrument for assessing self-perceptions of aging. BMC Geriatrics, 2007.

BRETAS, A.C. Cuidadores de idosos e o Sistema Único de Saúde. Revista Brasileira de Enfermagem. v.56, n.30, p.298-301, 2003. 
CÓTICA, Carolina Santin. Percepção de envelhecimento e finitude no final da vida adulta tardia: um estudo num grupo da melhor idade. [dissertação]. Universidade de Brasília, Brasília-DF, 2011.

CRESS, M.E.; BUCHNER, D.M.; QUESTAD, K.A. Exercise: effect sonphysical functional performance in independent older adults. Journal of Gerontology Advanced Biological Sciences Medical and Science, v.54, n.5, p.242-248, 1999.

DE VITTA, A. Atividade física e bem-estar na velhice. In: A.L. Neri; S.A. Freire. (orgs.). E por falar em boa velhice. Campinas-SP: Papirus, p.25-38, 2000.

DUTRA, S.N.L.P. Longevidade: a percepção do jovem e o seu próprio processo de envelhecimento. [dissertação]. Universidade Católica de Brasília, Brasília-DF, 2015.

FECHINE, B. R. A.; TROMPIERI, N. O processo de envelhecimento: as principais alterações que acontecem com o idoso com o passar dos anos. Revista Científica Internacional, ed.20, v.1, 2012.

GODOY, A. S. Introdução à pesquisa qualitativa e suas possibilidades. Revista de Administração de Empresas. São Paulo, v. 35, n. 2, p. 57-63, mar./abr, 2005.

HANSSON, R. O.; STROEVE, M. S. Integrating aging and bereavement in late life. In: HANSSON, R. O.; STROEVE, M. S (Eds.). Bereavement in late life: Coping,adaptation, and developmental influences. Washington, DC US: American Psychological Association, p. 153-172, 2007.

KAUFFMAN, T.L.; JACKSON, O.O. Indivíduo como um todo. In: KAUFFMAN, T.L. Manual de Reabilitação Geriátrica. Rio de Janeiro: Guanambara Koogan, 2001.

IBGE - Instituto Brasileiro de Geografia e Estatística. Censo 2010. Disponível em: http://cidades.ibge.gov.br/xtras/perfil.php?lang=\&codmun=293330\&search=\|infogr\%E1ficos :-informa\%E7\%F5es-completas. Acesso em: 06 de março de 2016.

MORAES, E.N.; et al. Princípios básicos de geriatria e gerontologia. Belo Horizonte: Coopmed; 2009.

MOTTA, L.B. Processo de envelhecimento. In: A.L. Saldanha; C.P. Caldas (Ed.). Saúde do Idoso: a arte de cuidar. 2a edição. Rio de janeiro: Interciência, p.115-124, 2004.

NERI, A.L. O fruto da sementes: processos de amadurecimento e envelhecimento. In: NERI, A.L. (Org.). Maturidade e Velhice: Trajetórias individuais e socioculturais. Campinas-SP: Papirus, 2001.

NETTO, M.P. História da velhice no século XX: Histórico, definição do campo e temas básicos. In: FREITAS, E.V.; L.Py.; NÉRI, A.L.; CANÇADO, F.A.X; GORZONI, M.L.; M.L e ROCHA, S.M. (Eds.). Tratado de geriatria e gerontologogia. Rio de Janeiro, Guanambara Koogan, p.-2, 2002. 
PASCHOAL, S.M.P. Epidemiologia do envelhecimento. In: PAPALÉO NETTO, M. Gerontologia. A velhice e o envelhecimento em visão globalizada. São Paulo: Atheneu, 2002.

RIBEIRO, L. da C. C.; ALVES, P. B.; MEIRA, E. P. Percepção dos idosos sobre as alterações fisiológicas do envelhecimento. Ciência, Cuidado e Saúde, v.8, n.2, p.220-227, 2009.

RIBEIRO, M. dos S.; CENDOROGLO, M.S.; LEMOS, N. de F.D. A percepção dos idosos acerca de seus hábitos de vida e comportamento de autocuidado quando jovens e a influência destes na saúde e envelhecimento. Revista Kairós Gerontologia, 18 (2), p. 81-10, 2015.

SHIN, K.; KIM, M.; KIM, Y. Study of the aging perception. Nursing \&Health Sciences, v.4, n.3, A8, 2002.

SILVA, E.M..; SILVA FILHO, C.E.; FAJARDO, R.S.; FERNANDES, A.U.R.; MARCHIORI, A.V. Mudanças fisiológicas e psicológicas na velhice relevantes no tratamento odontológico. Revista Ciência em Extensão, v.2, p.70, 2005.

SHEPHARD. R.J. Envelhecimento, atividade física e saúde. São Paulo: Phorte, 2003.

VERAS, R. A era dos idosos: os novos desafios. Anais da I Oficina de Trabalho sobre desigualdades sociais e de gênero em saúde do idoso. Ouro Preto-MG. p.89-96, 2002

YASSINE, I. M. C.. A auto percepção do envelhecimento e os traços de personalidade em idosos. [dissertação]. Universidade de Lisboa, 2011.

Como citar este artigo (Formato ABNT):

FILADELFO, J.S.; CÂNDIDO, A.S.C.. Percepção do Adulto-Jovem sobre o Envelhecer. Id on Line Revista Multidisciplinar e de Psicologia, Out-Nov. de 2016, vol.10, n.31, Supl 3, p. 172-183. ISSN 1981-1179.

Recebido: 31/10/2016

Aceito: 01/11/2016 